\title{
UN DIÁLOGO EN EL MUNDO DEL OTRO
}

\author{
Javier F. Nadal R.* \\ https://orcid.org/0000-0001-5319-8071
}

RECIBIDO: Mayo 2020 / ACEPTADO: Septiembre 2020 / PUBLICADO: Enero 2021

Como citar: Nadal, Javier. (2021). Un diálogo en el mundo del otro. Telos: revista de Estudios Interdisciplinarios en Ciencias Sociales, 23 (1), Venezuela. (Pp.27-35).

DOI: www.doi.org/10.36390/telos231.03

\section{RESUMEN}

El documento tiene como objetivo abordar las implicaciones en el diálogo entre el Otro y la Modernidad. Donde el lenguaje destaca como elemento primordial en la experiencia de la vida y que devela la historicidad de ese otro mundo, oculto en la palabra no dicha. Un lenguaje que traducido como elemento de poder ante la resistencia, siendo este, una pieza importante de enarbolar para abordar y enfrentar los mecanismos de la Modernidad. Este documento forma parte de un proyecto de investigación en curso, y entre los autores citados para apoyar este análisis se encuentra Ngugi wa Thiong'o (2015), Enrique Dussel (2008) y Ramón Grosfoguel (2007). Una vez hecho el análisis, es determinante la necesidad que existe de visualizar las subjetividades en un mundo que aún sigue siendo negado. Debido a que, en la actualidad, el accionar ante el diálogo franco requiere canales apropiados, no vislumbrados. Es necesario pasar de inmediato, de la teoría crítica a una práctica real contundente, que drene en las capas más profundas de la periferia.

Palabras claves: Lugar de enunciación; sujeto de enunciación; lenguaje.

\section{A dialogue in the world of the Other}

\begin{abstract}
The document aims to address the implications in the dialogue between the Other and Modernity. Where language stands out as a fundamental element in the experience of life and that reveals the historicity of that other world, hidden in the unspoken word. A language that is translated as an element of power in the face of resistance, this being an important piece to raise to tackle and confront the mechanisms of Modernity. This document is part of a research project and the main authors include Ngugi wa Thiong'o (2015), Enrique Dussel (2008) and Ramón Grosfoguel (2007). Once the analysis was done, I emphasize the need to visualize subjectivities in a world that is still being denied. For today, acting in the face of frank dialogue requires controlled channels that
\end{abstract}

* Universidad Nacional Experimental de las Artes. Correo electrónico. nadal001@gmail.com 
are not in sight. It is necessary to pass immediately, from critical theory to a forceful real practice, which drains into the deepest layers of the periphery.

Key words: Place of enunciation; subject of enunciation; language.

\section{Introducción}

El abordaje se realiza desde la postura de la transmodernidad, lo cual permite abrir un canal para la denuncia, de hechos que ocurren desde hace siglos. La opresión de los pueblos autóctonos y de grupos minoritarios, deja entrever las diferentes jerarquías que excluyen a los hombres y mujeres cosificados, como productos de la Modernidad. Una realidad que cultiva subjetividades, erige al Otro, marcado por el silencio, la dominación y el despojo. En un discurso hegemónico que niega su historicidad y ser.

En tal sentido, el diálogo siempre ha debido entablarse en el mundo del Otro, sin la pretensión de alterarlo, reconociéndole como ser, apreciar su lengua como medio de comunicarse con la esencia de cosmos, sus pares y consigo mismo.

Un proceso importante es activado con el surgimiento de las teorías críticas, y es que a partir de ellas, inicia el reconocimiento de las voces colonizadas, de quién habla sobre el proceso de colonización, de sus consecuencias, así como la necesidad de develar el mito de la modernidad y el antagonismo que ha traído su lado más nefasto, el colonialismo.

Considerar el proceso de ocultamiento del Otro, es reconocer su existencia en un mundo humano, develarlo a la luz de una ética que consagre su esencia ontológica y epistemológica. Sin embargo, la realidad, es que, yace en un mundo en el cual es violentado y silenciado, donde no le es permitido ser.

En virtud de ello, el objetivo de este documento es abordar las implicaciones en el diálogo entre el Otro y la Modernidad. Donde el lenguaje destaca como elemento primordial en la experiencia de la vida y que devela la historicidad de ese otro mundo, oculto en la palabra no dicha. En este, se aborda el leguaje como elemento de poder y la necesidad de la recolocación del locus de enunciación. A su vez, se articula con el silencio que se gesta de mano de la Modernidad. Por lo que se entabla la importancia del lenguaje dentro de la cultura y como elemento de producción, al vislumbrar nuevas perspectivas en torno a ese diálogo.

\section{Desarrollo}

La voz que cuenta el proceso de colonización, esta se sitúa, inicialmente y de manera exclusiva, en el Primer Mundo. Por supuesto, lo que expresa, está supeditado al sentir del colono, bajo la óptica moderna. De allí que, no existe consecuencia alguna que pueda criticarse a este proceso (Mignolo, 2005). Al contrario, era gestado según los parámetros esperados.

Por lo que, son las voces de los países que representan ese Primer Mundo, las que enuncian, desde donde el capitalismo tiene su génesis y los que instauran las categorías propias de la racialización y quienes actúan bajo una presunta democracia (Mignolo, 2005).

En virtud de ello, es necesario precisar que, las voces erigidas están asociadas a posiciones políticas, no geográficas. Acción activada al tratar Europa de elevar su racionalidad como primicia, y subsumir otras formas de pensamiento bajo su perspectiva moderna. Colocándose como una cultura salvadora del resto del mundo. 


\section{Donde se ubica la enunciación}

He aquí un primer momento, era reconocido el lugar donde estaba ubicaba la enunciación y las razones del por qué operaba desde allí. Ahora era necesario, retomar esa voz, y es hacia donde apuntan las teorías críticas. Al respecto, Mignolo (2005) considera que, este acto, es convertido en una batalla, al tratar de desplazar el lugar de enunciación hacia el tercer mundo. Un tercer mundo inventado, según Mignolo (2014) "por hombres e instituciones, lenguas y categorías de pensamiento del Primer Mundo" (p.31).

Es necesario el posicionamiento del locus desde donde surge la enunciación, porque hasta ahora según Ribeiro (2019), "aún no puede hablar, el niño sin voz propia, a quien nos referimos en tercera persona, porque habla por medio de la interpretación de los adultos" (p.15). Pero ese Otro, al hablar, tiene mucho que decir, posee incontables demandas. Ante esta perspectiva, es el momento, no puede haber prórroga. Es la oportunidad de expresar lo reprimido, deben colocarse las peticiones y trazar su ruta con nuevos y propios designios libertarios.

Y quienes están llamados a tomar posturas en contra de lo sufrido, según Mignolo (2005), son "aquellos para quienes las herencias coloniales son reales (ej., les duele) están más (lógica, histórica, y emocionalmente) inclinados que otros a teorizar el pasado en términos de historias coloniales" (p.8). Y extender la invitación a otros que desde diferentes latitudes, acompañen ante esta enunciación, identificados al criticar, la manera bestial con que los pueblos fueron ultrajados. Esto ratifica la urgencia, no solo de tomar posesión, sino de estimular "la constante producción de lugares diferenciales enunciación" (Mignolo 1995, p.32).

Al respecto, plantea Ribeiro (2019), "Pensamos lugar de enunciación como una forma de refutar la historiografía tradicional y la jerarquización de saberes consecuente de la jerarquía social" (p.17). Y parte de esa demanda es desmantelar la premisa de elevar el saber europeo como único, y desestimar el resto de las culturas del mundo, así como revalorar la historiografía de esos pueblos. En virtud, afirma la autora que, al referir "del Lugar de Enunciación estamos hablando de locus social, es decir, del lugar social desde donde los grupos se originan" (p.17).

Tal responsabilidad vislumbrada en lo que Mignolo (1995) considera al "Construir nuevos Lugares legítimos de enunciación que hagan posible la re-locación de las construcciones imaginarias" (p.39). Esta relocalización es una deuda con los ancestros de todas las sociedades trastocadas por la Modernidad, incluso, por las generaciones por venir. Esta construcción requiere según Ribeiro (2019), "romper con el régimen de autorización discursiva, con el silenciamiento y/o supresión de saberes, para combatir la violencia estructuralmente impuesta contra grupos oprimidos" (p.17). Lo que implica, tomar el mando a la hora de alzar la voz, apreciar cada oportunidad y no vacilar al hacerlo. Revalorar a estos pueblos, a los grupos oprimidos implica, reconocer el valor de su voz, de su ser, significa construir canales de dialogo para la emancipación.

Al respecto, plantea Jiménez (2013), "en América Latina, se han realizado rupturas, digamos epistemológicas, pero también políticas, que entre otras cosas, se relacionan con un desplazamiento del "locus" de enunciación que consiste en no pensar, ni escribir desde los grandes centros civilizatorios de occidente" (p.3). Por consiguiente, es preciso cimentar "nuevos y diversos lugares desde los cuales, pensar y enunciar con nuevos "olores, colores y sabores" (Jiménez 2013: p.3). Lo que conduce a una postura que asuma el desafío, que implica escribir lo que somos, del valor de lo autóctono. Elevar nuestra historia y construir el legado arrebatado. 


\section{La razón es quien silencia}

En función a lo planteado, es necesario a su vez, precisar la necesidad que hay de privar al "bárbaro" del reclamo. Y es que, ante la violencia contra "el Otro", es necesario justificar la acometida inhumana. A través del invasor que posibilita que una civilización subsuma a los pueblos, dando así origen a una categoría diferencial entre pueblos civilizados y no civilizados. Ante esa apertura, bastaría ahora, observar, detenerse a conceptualizar las premisas que justificarían su hecho. Y para ello, entre lo apreciado, según Neila (2009), "Primero los exploradores y luego los misioneros y administradores hallaron una satisfactoria comprobación de las teorías sobre el primitivismo, sobre la ruralidad y sobre la práctica indistinción entre sociedad humana y naturaleza" (p.46).

Estos vieron en la expresión oral indígena, las señales de "imperfecciones incivilizadas" (Neila, 2009, p.46). Las fallas que deseaban precisar, eran mostradas. Para los conquistadores, según este autor, el empleo de la oralidad condensaba, todo el atraso civilizatorio que podía tener un pueblo.

Y al no registrar su historia, su dinámica, a través de la escritura, serán considerados no civilizados. Puesto que, no evolucionan como la cultura occidental. Según Roca e Iniesta, citados por Neila (2009). Y cómo podrían, al no tener registros de su pasado, cómo lograrían poder hacerse de un porvenir. Esto en efecto, ya que según la conciencia europea, carecían del saber del pasado, al no registrar su historia, y encontrase confinados en el lenguaje oral.

Sin embargo, Dorra, citado por López (2017), sostiene "que el propio concepto de "oralidad" es en sí mismo una noción occidental (...), al hablar de oralidad nos situamos de hecho en el espacio de la escritura" (p.200).

Desde esta perspectiva, es una construcción empleada, con el propósito de banalizar al Otro. Condenar la oralidad, permitiría asociar el hacer indígena a lo tradicional. Para hacerlo ver, alejado del conocimiento racional, por lo que el "insipiente saber" censura incluso, su evolución. Sin embargo, enfrentar lo oral con lo escrito, implica separar las correspondencias históricas generadas en ambos campos (López, 2017).

En virtud de ello, el notorio énfasis en este aspecto, al describir a los pueblos originarios, según Neila (2009), y concluir que estos estaban basados en la tradición no en la historia. Estos "pueblos primitivos" eran encontrados al margen de ser considerados seres con historia. Y su herencia conductual era vinculada al instinto e imitación.

Según los objetivos propuestos, había que acabar con esa tradición y con el mecanismo que las activaba, la lengua. Ya que según Ngugi (2015), "La lengua, cualquier lengua, tiene un carácter dual: es simultáneamente un medio de comunicación y un vehículo de la cultura humana" (p.54). Aunque inicialmente fue banalizado, este proceso natural y humano, habría que detenerlo.

Desde esta perspectiva, habría la necesidad de contenerle, en virtud a que este proceso brinda "la capacidad de hablar, (...) la comprensión mutua entre los seres humanos" (Ngugi, 2015, p.60). Era necesario encubrir, hasta el punto de silenciar, ya que según Fanon (2009), ese lenguaje expresa el mundo del hombre y a su vez, las implicaciones de lenguaje en el contexto.

Y es que, para Varese (2018), "El lenguaje cultural indígena está construido alrededor de unos cuantos principios y una lógica cultural o topología cultural que privilegia la diversidad 
(...) y la heterogeneidad sobre la homogeneidad, el eclecticismo sobre el dogma, la multiplicidad sobre la bipolaridad" (p.28). Es decir, este lenguaje amplía la concepción del cosmos, estaba relacionado con su todo. Una concepción que, en un primer momento, solo el indígena conocía.

Por lo que, vulnerar la lengua del nativo, en definitiva, era perentorio, y el primer paso, era sometiéndole con otra lengua, la del colono. Esta acción era de tal significación, en tanto que, a través de la lengua es posible doblegar al hombre, está por sí misma, posee gran fuerza, al punto de sumergir en la docilidad a su alma (Ngugi, 2015).

Al respecto, es necesario considerar lo planteado por Fanon (2009), cuando afirma que hablar denota "asumir una cultura, soportar el peso de una civilización" (p.49). Y es otro aspecto a razonar, ante el daño ejercido en el otro. Cuando es subsumida su cultura, mientras debe admitir otra, que le doblega y humilla.

Llevar a cabo esta sustitución considera Neila (2009), es un proceso que va "en detrimento de las lenguas autóctonas. Un aspecto fundamental en la construcción de la identidad." (p.54). Así, era construía el proyecto de la Modernidad, configurando el individuo necesario para su desarrollo. Gracias a los mecanismos modernos, a su gestión civilizadora y el empleo de la lengua colonial, se articula lejos de la nación colonizadora (Neila, 2009).

Bajo esta perspectiva, aceptar la lengua no originaria, a la fuerza, era según Mignolo (2014) "aceptar la humillación de ser inferior a quienes decidían que debías mantenerte como inferior $\mathrm{o}$ bien, asimilarte. $Y$ asimilarte significa aceptar tu inferioridad y resignarte a jugar un juego que no es tuyo, sino que te ha sido impuesto" (p.29).

Aunado a ello, plantea Ngugi (2015), otro aspecto que había que borrar era la cotidianidad del indígena, habría que brindarle las connotaciones europeas, elementos importantes a la hora de situarlos como colonia. Y el habla nativa tenía en su seno el lenguaje real de su vivir. De allí que, en ella permanecía su esencia de pueblo. Estas lenguas mostraban un impedimento del proceso civilizatorio, contenían, expresa Ngugi (2015), "sus propias bases sociales y culturales, y estas son instrumentales en la formación de los procesos mentales y los juicios de valor" (p.239). De allí que, era necesaria su mutilación.

En la actualidad, considera Grosfoguel (2007), que "La epistemología eurocéntrica hegemónica asume un punto de vista universalista, neutral y objetivo. Nadie escapa a las jerarquías de clase, raciales, sexuales, de género, lingüísticas, geográficas y espirituales del sistema-mundo euro/norteamericano capitalista/patriarcal moderno/colonial" (p.3). En este sentido, el Otro, piensa y siente desde las voces de la superioridad, aún desde las voces de los colonos. Es decir, que al usar sus reglas, sus métodos, los pueblos colonizados, solo son parte de su sistema que privilegia de un sistema global, donde "los "otros" producen religión, folklore, mitos, pero nunca teoría de conocimientos" Grosfoguel (2007, p.8).

Sin embargo, plantea Neila (2009), la impetuosa necesidad de "La relocalización de lenguas, pueblos y conocimientos en el tiempo (...) que permanecieron incontestadas hasta hace unos cincuenta años, cuando los intelectuales comprometidos con los movimientos de liberación y descolonización pusieron en duda sus premisas" (p.55). En este contexto, no hay que subestimar ninguna acción, por pequeña que sea, siempre que apunte en el levantamiento de estas voces. 


\section{El sujeto de enunciación}

Por lo cual, es preciso enfatizar en lo planteado por Grosfoguel (2007), sobre "el lugar epistémico de enunciación, esto es, la localización geopolítica y corpo política del sujeto que habla/enuncia en las coordenadas del poder global" (p.3). Es decir que, bajo la perspectiva decolonial, existe una necesidad de ubicar al sujeto que enuncia desde el enfoque epistémico, cuyo pensamiento lo ubica en la periferia.

En relación a este aspecto, es un patrón característico que "En la filosofía y las ciencias occidentales el sujeto que habla siempre queda escondido, encubierto y borrado del análisis" (Grosfoguel, 2007, p.3). Este comportamiento, articulado con el fin de objetivar el proceso, permite anular la subjetividad.

En virtud de lo planteado, expresa Grosfoguel (2007): la epistemología occidental "esconde a quien habla y la localización epistémica en las relaciones de poder desde la cual el sujeto habla" (p.4).

Más allá de esta afirmación, Borsani y Quintero (2014), sostienen que, a lo que hay de darle importancia no es precisamente a "la enunciación sino el sujeto enunciante, (...) quién dice qué, desde dónde, (...) con qué intencionalidad, qué lenguas, (...) que historias (locales) están involucradas" (p.16).

De cara a lo planteado, es preciso que no haya incertidumbre para reconocer la historicidad del enunciante. Para develar a este individuo, es necesario conocer la metodología con que opera el proceso de la Modernidad, con su "naturalidad" esconde siempre su intención en pro de la colonialidad. Por lo que Quijano, citado por Grosfoguel (2007), expresa "todavía vivimos en un mundo colonial y necesitamos una ruptura anti-sistémica descolonizadora con las maneras estrechas y limitadas de pensar las relaciones coloniales" (p.13).

Bajo esta tendencia, una pieza característica del accionar moderno, es su postura de superioridad, desde donde ejerce su poder, imponiendo "Los diseños globales monolingüistas y monotópicos de Occidente" (Grosfoguel, 2007, p.14). A su vez, penetra diferentes culturas invisibilizando las cosmologías y epistemologías de estos (Grosfoguel, 2007). Puesto que solo hay una perspectiva válida, relegando los puntos de vista que identifica a las minorías, estas no perfilan como una voz significativa para ser considerada epistémicamente válida.

Hasta ahora, el discurso de la argumentación no cristaliza, aunque la violencia es justificada. El mecanismo para incorporar al Otro a la comunidad de comunicación, es otro artificio la Modernidad, Dussel (2008). Todo quedó en un discurso fraudulento, traducido en silenciar y no escuchar.

Lo anterior se traduce, según Dussel (2008), de "un diálogo entre "la razón del Otro" y el "discurso de la Modernidad" naciente. No había simetría (...) ya que unos eran los vencidos, y otros los vencedores" (p.124). Y por ende, era precisa una mayor apertura para el logro del entendimiento. Puesto que, el supuesto diálogo, era promovido desde una lengua "hegemónica, la que tenía el poder" (Dussel, 2008, p.125).

De aquí que, para constituir un diálogo franco, era preciso abrirse al mundo del Otro, y aunque no sería fácil y nada amplio el camino a transitar, tal acción, permitiría abrir canales para su comprensión. Esta operación inmediata, requería el "conocer su lengua", el mensaje de sus palabras y el significado de estas en sus vidas (Dussel, 2008). Era una decisión trascendental, pero elegía una vía violenta. 


\section{El lenguaje como elemento de producción}

Al respecto, en la comunidad indígena, sus relaciones estaban centradas en francos diálogos, ejemplo que daban los mismos sabios de las tribus, (Dussel, 2008). El entablar conversaciones era matriz de su accionar. Pero aquí "los indios estaban como mudos y los españoles como sordos" (p.124). Constituyéndose la primera, en una sordera hegemónica, según lo acuña Palermo (2014). Por lo que, entre la Modernidad y el Otro, no hubo una plática.

Un aspecto significativo también lo es que, al silenciar la voz del Otro y sustituirlos por códigos foráneos, es que el lenguaje como manifiesto de relación, es una pieza importante en la producción. En virtud que, dinamizan los procesos a través de los cuales las personas establecen los medios para vivir (Ngugi, 2015). Un perfil que aborda el factor económico sobre el cual la Modernidad descansa. De allí, la necesidad de subsumir al individuo, convirtiéndole en una cosa que produce.

En este sentido, establecerse en el Otro a través de su lenguaje, es empoderarse del emprendimiento en el trabajo, y la expansión que hace de ello con el resto de la comunidad. Lo que lleva a la producción de fortuna, alimento y habitación. Estas relaciones son propias del monopolio moderno (Ngugi, 2015).

Hasta ahora, el proyecto estaba claro, sin embargo, el propósito del Occidente sigue su camino. Aún en estos días, esas voces reclaman atención, por lo que plantea, Arundhati Roy, citado por Ngugi (2015), que "Existen solo las deliberadamente silenciadas, y a quienes preferimos no escuchar" (p.14).

No obstante, en la actualidad, el panorama no ha cambiado, el Otro aún tienen mucho que demandar, de las condiciones infrahumanas en la que labora el obrero, la explotación del campesino, de la ama de casa, del indígena. Entonces, "Alguien tiene que hablar a gritos sobre las condiciones laborales" (Ngugi, 2015, p14). Sobre sus condiciones deplorables de vida que, aún persisten.

\section{Nuevos perspectivas ante el dialogo}

En este sentido, afirma Dussel (2008), las acciones deben conducirse a potenciar la "teoría" o "filosofía del diálogo" -como parte de una "Filosofía de la Liberación" del oprimido, del incomunicado, del excluido, del Otro-, sobre las condiciones de posibilidad histórica hermenéutica de la "comunicación" intercultural" (p.10). Articulaciones que permitan visibilizar al individuo silenciado, cosificado, humillado, al condenado de la tierra de Fanon.

Al respecto, Palermo (2014), lanza una invitación, hay que hacer teoría, pero desde otro lugar. Hay que hacerlo desde la calle, del barrio. Buscar allí nuevos códigos y categorías, a partir del lenguaje popular impregnado de su cultura, de lo local.

Bajo esta perspectiva, es necesario profundizar en un giro decolonial, según Veronelli (2015), de cara a un paradigma del lenguaje que: "conlleva la lógica de la colonialidad, porque prescribe una relación entre lenguaje y poder; lenguaje y territorio; lenguaje y escritura" (p.36).

En tal sentido, afirma Meza (2017), que aunque "la humanidad no ha tenido una misma visión e interpretación del mundo, no ha tenido un mismo lenguaje" (p.11). De aquí, la necesidad de buscar puntos de encuentro, inflexiones que permitan una comunicación sólida para emprender acciones conjuntas.

Ante lo que Palermo (2014), propone como la posibilidad de "iniciar un diálogo trans/intercultural con los movimientos sociales, que generan sus propias alternativas, para 
avanzar hacia una práctica democrática decolonial al propiciar la puesta en acto de lo que Mignolo denomina una "hermenéutica pluritópica" (p.145).

Según estas perspectivas, uno de los rangos de acción debe ir encaminado hacia los individuos a quienes aún hoy día les es vulnerada la vida, hay que ir más allá de identificar ese lugar de enunciación de sí mismo y favorecer a la constructividad del individuo, su recomposición (Escobar, 2003).

\section{Conclusión}

Es necesario admitir que el diálogo en el mundo del otro, no está constituido. En América Latina, las estructuras de la Modernidad enquistadas en estos países y en sus gobiernos, no permiten esta acción. Los canales apropiados no existen. Un clima marcado por la corrupción, el populismo, la desidia, la racialización y muerte, socaban al hombre y a la mujer que aspiran, alguna vez, ser escuchados. Mientras la pobreza merma sus sueños, el hambre no les permite dormir. Al tanto que, ven sus utopías evaporarse, desterrados de su propio ser. Hoy, todavía son cosificados, viviendo un infierno donde el calor les surca la piel. Irónicamente allí, la razón también silencia.

Por lo que es necesario pasar de inmediato, de la teoría crítica a una práctica real y contundente, que drene las capas más profundas de la periferia, ya que en el infierno no existe el diálogo, solo hay lamentos.

\section{Referencias bibliográficas}

Borsani, María y Quintero, Pablo (2014). Los desafíos decoloniales de nuestros días: pensar en colectivo. En María Borsani y Pablo Quintero. Los desafíos decoloniales de nuestros días: pensar en colectivo. Neuquén: EDUCO. Argentina. Disponible en: http://www.ceapedi.com.ar/imagenes/biblioteca/libreria/332.pdf

Dussel, Enrique (2008). 1492: El encubrimiento del otro. Hacia el origen del mito de la modernidad. Biblioteca Indígena. Bolivia.

Fanon, Frantz (2009). Piel negra, máscaras blancas (segunda edición). Lavel S.A. España.

Grosfoguel, Ramón (2007). Implicaciones de las alteridades epistémicas en la redefinición del capitalismo global: transmodernidad, pensamiento fronterizo y colonialidad global. En Mónica Zuleta, Humberto Cubides y Manuel Escobar. ¿Uno solo o varios mundos? Diferencia, subjetividad y conocimientos en las ciencias sociales contemporáneas. Siglo del Hombre Editores. Colombia. Disponible en: http://books.openedition.org/sdh/416>

Jiménez, Jonathan (2013). Desplazamiento del locus de enunciación: desde la tierra de las visitas inoportunas. Revista de Teología. Vol. 7 Núm. 2. Costa Rica. (Pp.97-119) Disponible en: https://www.revistas.una.ac.cr/index.php/siwo/article/view/6279

López, Alejo (2017). Literatura niuyorriqueña y corpo-política: el cuerpo como núcleo de significación poética. Palimpsesto Vol. IX, Núm 12. Chile (Pp.196-216). Disponible en: http://www.revistas.usach.cl/ojs/index.php/palimpsesto/article/download/2989/2722/

Meza, Guillermo (2017). Ética de la investigación desde el pensamiento indígena: derechos colectivos y el principio de la comunalidad. Bioética y Derecho. Núm.41 España. (Pp.141-159) 
Mignolo, Walter (1995). Occidentalización, Imperialismo, Globalización: Herencias Coloniales y Teorías Postcoloniales. Revista Iberoamericana. Vol. LXI, Núm. 170-171.Estados Unidos. (Pp.27-40). Disponible en: http://revistaiberoamericana.pitt.edu/ojs/index.php/lberoamericana/article/viewFile/6392/6568

Mignolo, Walter (2005). La razón postcolonial: Herencias coloniales y teorías postcoloniales. AdVersuS. Volumen II, $\mathrm{N}^{0} 4 . \quad$ Argentina. Disponible en: http://www.adversus.org/indice/nro4/articulos/articulo_mingolo.htm

Mignolo, Walter (2014). Retos decoloniales, hoy. En María Borsani y Pablo Quintero. Los desafíos decoloniales de nuestros días: pensar en colectivo. Neuquén: EDUCO. Argentina.

http://www.ceapedi.com.ar/imagenes/biblioteca/libreria/332.pdf

Neila, José (2009). La descolonización de las mentes en el África Subsahariana: identidad y conocimiento social. Revista Estudios Internacionales. Vol. 41 No. 162. Chile. (Pp. 31- 62). Disponible en: https://revistaei.uchile.cl/index.php/REl/article/view/13736

Ngugi wa Thiong'o (2015). Descolonizar la mente. La política lingüística de la literatura africana (cuarta edición). Penguin Random House Grupo Editorial, S. A. U. España.

Palermo, Zulma (2014). Irrupción de saberes "otros" en el espacio pedagógico: hacia una "democracia decolonial". En María Borsani y Pablo Quintero. Los desafíos decoloniales de nuestros días: pensar en colectivo. Neuquén: EDUCO. Argentina. Disponible en: http://www.ceapedi.com.ar/imagenes/biblioteca/libreria/332.pdf

Ribeiro, Djamila (2019). Breves reflexiones sobre Lugar de Enunciación. Relaciones Internacionales. No. 39. México. (Pp. 13- 18). Disponible en: https://doi.org/10.15366/relacionesinternacionales2018.39.001

Varese, Stefano. (2018). Los fundamentos éticos de las cosmologías indígenas. Amérique Latine Histoire et Mémoire. Les Cahiers ALHIM. Núm.36. Disponible en: https://journals.openedition.org/alhim/6899

Veronelli, Gabriela (2015). Sobre la colonialidad del lenguaje. Universitas humanística. Núm.81. Colombia (Pp. 33-58). Disponible en: http://www.scielo.org.co/pdf/unih/n81/n81a03.pdf 